\title{
Ética militar e inteligencia artificial: reflexiones para Colombia desde el contexto global actual ${ }^{1}$
}

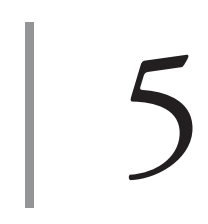

https://doi.org/10.21830/9789585377134.05

\author{
Cristian Gantiva Castiblanco ${ }^{2}$ \\ Escuela Superior de Guerra "General Rafael Reyes Prieto"
}

\section{Resumen}

El objetivo de esta investigación es plantear las reflexiones que deben hacer las Fuerzas Militares de Colombia sobre los límites éticos de la inteligencia artificial (IA) con base en el contexto global actual. Las propuestas realizadas obedecen a un método cualitativo de revisión documental relacional, en el que se optó por un alcance exploratorio y descriptivo sobre la información relacionada con IA y ética, IA y defensa e IA, ética y defensa. En los resultados se identifican a grandes rasgos las formas en que se han abordado estos temas, el nivel de enraizamiento y densidad de ciertas categorías, subcategorías y características en la literatura y, por último, algunos elementos emergentes de importancia para destacar. Finalmente, con base en el análisis se hacen sugerencias necesarias que el sector defensa en Colombia, especialmente desde las Fuerzas Militares, deben comenzar a revisar.

Palabras clave: defensa; cibernética; ciencia y sociedad; ética; ética militar; inteligencia artificial.

1 Este capítulo presenta los resultados colaborativos de dos proyectos de investigación: (1) "Ética militar e inteligencia artificial: reflexiones para Colombia desde el contexto global actual", del Grupo de Investigación Masa Crítica de la Escuela Superior de Guerra "General Rafael Reyes Prieto", Colombia, categorizado en B por Minciencias y con código de registro COL0123247, y (2) "Mujeres de arma, seguridad y defensa nacional. Un análisis desde sus percepciones", del grupo de investigación en Ciencias Militares, de la Escuela Militar de Cadetes "General José María Córdova", Colombia, categorizado en B por Minciencias y con código de registro COL0082556. Los puntos de vista pertenecen al autor y no reflejan necesariamente los de las instituciones participantes.

2 Magíster en Derechos Humanos y Derecho Internacional de los Conflictos Armados de la Escuela Superior de Guerra. Profesional en Ciencia Política y Gobierno de la Universidad del Rosario. ORCID: https://orcid.org/0000-0003-0367-8901 


\section{Introducción}

El interés por conocer y analizar los alcances de la inteligencia artificial (IA) a nivel mundial se encuentra en constante crecimiento y, de la misma forma, los avances científicos y tecnológicos a su alrededor; basta con buscar en Google Trends "inteligencia artificial” o "artificial intelligence" para verificar el rastreo e incremento exponencial de información sobre este tema, al menos durante los últimos diez años. Este hecho, y una mirada superficial sobre los resultados, también refleja, en cierta medida, la multiplicidad de abordajes desde todas las ciencias formales y no formales y, a partir de ahí, la complejidad en consolidar paradigmas, posturas y propuestas homogéneas sobre la trascendencia de la IA en las dinámicas sociales, económicas, políticas, culturales y militares.

Uno de los debates y subtemas más álgidos en ese gran mundo de información, en el que Colombia se ha incorporado de modo activo a partir del año 2020 con el Consejo Nacional Político, Económico y Social - Conpes 3995 y el documento para discusión presentado en agosto de ese ańo como el "Marco ético para la inteligencia artificial en Colombia”, trata sobre las implicaciones éticas de todo aquello que conforma la IA. El desarrollo de esta discusión es tan amplio como la posibilidad misma de la IA en cualquier esfera o nivel de la vida humana, sin embargo, de manera clara, por su naturaleza, hay al menos cuatro sectores que sobresalen de los demás: salud, defensa, justicia y gobierno. Esta investigación, aunque examina el tema de manera general, se centra en una mirada para el sector defensa y la perspectiva ética puntual que requieren las Fuerzas Militares.

La ética, de por sí, ha sido extensamente discutida de forma teórica y conceptual. Como argumenta Robles Carrillo (2020) se encuentran al menos tres factores de inflexión, vacío y debate que no permiten la existencia o configuración de una definición única de un "código de ética universal": la historia, la geografía y lo material (pp. 2-3). Este complejo análisis que, de forma innata, se plantea profundo e inagotable sobre las decisiones que toman los seres humanos, se torna vago e indeterminado cuando se extrapola a decisiones basadas en algoritmos y procesamientos automatizados de información 
y, mucho más, cuando estas decisiones implican una posible afectación directa o indirecta sobre los derechos fundamentales de las personas en el cumplimiento de misiones y funciones institucionales.

Resulta elemental para los Estados y las sociedades, en el marco de este escenario, comenzar a sentar una posición jurídica, pero también cultural, desde el punto de vista de la responsabilidad, que conceda claridad sobre las formas en que se da la vinculación de la innovación científica y los humanos en los últimos tiempos. Floridi et al. (2018) proponen al menos cuatro preguntas que guíen esa vinculación: ¿quiénes podemos llegar a ser?, ¿qué podemos hacer?, ¿qué podemos lograr? y ¿cómo podemos interactuar con otros y con el mundo? Para el caso de esta investigación, las cuatro preguntas son esenciales, considerando que la IA en la defensa, como se verá más adelante, puede llegar a ser crítica en la ilación y uso en operaciones de las Fuerzas Militares; de ahí que, por ejemplo, una de las áreas estratégicas de la investigación y el desarrollo en IA de los Estados Unidos sea "entender y abordar las implicaciones éticas, legales y societales de la IA" (Executive Office of the President of the United States, 2019, p. ii).

Así, considerando las distinciones presentadas en los párrafos anteriores, el presente capítulo tiene la siguiente pregunta de investigación: ¿̨uáles deben ser las reflexiones sobre los límites éticos de la IA desde las Fuerzas Militares de Colombia tomando como referencia el contexto global actual? Para responderla, se propone de forma específica: (1) identificar reflexiones generales sobre los límites éticos de la IA; (2) demarcar los campos de aplicación de la IA en el sector de la seguridad y la defensa y (3) definir las reflexiones tempranas que deben hacer las Fuerzas Militares de Colombia sobre los límites de la IA.

Con el fin de estructurar el documento, primero se presenta el marco teórico y conceptual que permite definir lo que entendemos por ética, así como también las formas en que el concepto se relaciona con lo que conocemos como inteligencia artificial. Segundo, se describe el diseño metodológico realizado, que posibilita asimilar y replicar los procedimientos, las técnicas y el paradigma que permitieron obtener los resultados. Tercero, se exponen los resultados encontrados en orden de importancia por enraizamiento y densidad. Cuarto, se discuten los productos, estableciendo su relevancia orien- 
tada por la pregunta de investigación y resolviéndola. Asimismo, se comentan los hallazgos inesperados y la contribución del proceso al objeto que se indaga. Quinto, finalmente, se proponen las conclusiones retomando las contribuciones más significativas e identificando posibles líneas por seguir, tomando como punto de partida la presente investigación.

\section{Marco teórico-conceptual}

El concepto de ética ha sido profundamente estudiado y debatido, no solo de forma aislada desde las ciencias sociales y humanas y desde un fundamento antropológico, sino también, de forma abierta, desde los puntos de encuentro y aproximación con diferentes ópticas relacionadas con la acción humana. Por lo anterior, la revisión sobre el concepto puede tener diferentes acepciones y argumentaciones supeditadas a contextos sociológicos o históricos espaciotemporales que, sin embargo, para la elaboración de este marco teórico, que tiene el objetivo de plantear qué es la ética de la inteligencia artificial, se espera determinar de tres breves maneras: (1) el concepto de ética y la ética militar, (2) el concepto de inteligencia artificial y (3) el concepto de ética de la inteligencia artificial.

\section{Ética y ética militar}

La ética, según la Real Academia Española de la Lengua (2019), es un conjunto de normas morales que determinan la forma de actuar en diferentes aspectos de la vida humana. Mucho más allá de este concepto general, hay una rama de la filosofía que se ha dedicado a componer, descomponer y recomponer el concepto, generando debates a su alrededor, pues si bien la mayoría de las propuestas pretenden abarcar qué es la ética, se trata de un criterio subjetivo que cambia o puede modificarse a través del espacio geográfico, temporal o cognoscitivo.

La ética puede ser entendida como una forma de reflexión (Betancur, 2016) o, para Paredes y Velasco (2014), como una ciencia o disciplina, que también puede ser confundida o trastocada con la moral y lo legal. Para efectos de este capítulo, la ética es la ciencia que orienta y permite a los seres humanos, 
desde la introspección, definir las normas, acciones, comportamientos o actos morales. La moral, y la calificación de un comportamiento como moral, se relaciona con "lo bueno, lo justo, conveniente a una realidad social, costumbre o ley para dictaminar si es humanizadora [...] es la totalidad de las normas impuestas o la exigencia pesante e histórica del hombre" (Paredes \& Velasco, 2014, p. 11).

Ahora bien, como se comentó, la ética tiene diferentes formas de entendimiento e interpretación. En este trabajo se aborda la perspectiva militar de la ética desde algunas singularidades que sobresalen. De Swarte et al. (2019) establecen que la doctrina militar enfatiza que las elecciones éticas son cuestiones de valores, organizados por reglas militares y su jerarquía (p. 294). Para las Fuerzas Militares de Colombia (2016), esta apreciación se desarrolla, pero va más allá, teniendo en cuenta que la ética es la "ciencia directiva de los actos humanos hacia el bien honesto, de acuerdo con la recta razón" (p. 8). Se tienen, a manera de asociación, por un lado, como parámetros de alta calidad para la ética profesional: la moral, los principios, el valor y la virtud y, por otro lado, como características particulares de la profesión militar: la fe en Dios, la fe en la causa, la primacía de lo colectivo, la disponibilidad permanente, la disposición para defender la vida, el sacrificio generoso y la disciplina, la competencia profesional, el humanismo, el servicio y el carácter firme (Fuerzas Militares de Colombia, 2016, pp. 12-13).

\section{Inteligencia artificial}

La inteligencia artificial está, hoy en día, vinculada a otros conceptos que forman parte, la conforman y sin los cuales sería incompleto su entendimiento. Para iniciar, el concepto puede relacionarse con sistemas tecnológicos que actúan y piensan racionalmente y como humanos, algunos de los cuales se pueden denominar autónomos. No obstante, hay autores como Marín García (2019) que argumentan que el empleo de este término puede ser confuso, pues "hasta la fecha ningún sistema o artefacto inteligente es capaz de dar cuenta de sus propios actos y decisiones de la manera en la que las personas son capaces, [por lo cual] resulta erróneo calificar a estos dispositivos de autónomos" (p. 10). 
Por otra parte, la inteligencia artificial tiene una connotación científica, expresada en esfuerzos multidisciplinarios para desarrollar máquinas capaces de aprender, tomar decisiones y actuar de manera inteligente en cualquier ambiente (Russell \& Norvig; citados por Ferrario et al., 2020, p. 525). Este tipo de procesos necesariamente involucran diferentes formas de diseño, desarrollo, implementación y evaluación, como el aprendizaje de máquina (machine learning), el cual se relaciona en mayor medida con las dos primeras fases. El aprendizaje de máquina es una disciplina que combina la estadística, la big data y los algoritmos para crear sistemas capaces de generar predicciones automáticas, con base en el aprendizaje sobre datos preseleccionados o preestablecidos.

Determinadas perspectivas, fundamentadas en las neurociencias y las ciencias cognoscitivas, se han puesto a la tarea de conceptualizar la IA desde su comparación con la inteligencia humana. La principal diferenciación, según Ryan (2020), radica en la construcción artificial de la inteligencia de las máquinas que, como argumentan Jensen et al. (2020), es una actividad dedicada a hacer a las máquinas "inteligentes", concibiendo la inteligencia como una cualidad que permite a una entidad funcionar de manera apropiada con precaución en ciertos ambientes (p. 530). La Junta de Asesoramiento en Innovación de los Estados Unidos tiene un concepto similar de IA, y mucho más simplificado que tomaremos como guía para el capítulo: "Es una variedad de técnicas y tecnologías de procesamiento de información usadas para realizar un objetivo/tarea y los medios para razonar la realización de esa tarea” (Defense Innovation Board, 2020, p. 5).

\section{Ética de la inteligencia artificial}

Finalmente, valorando lo que representa la ética y la ética militar y el campo de acción general de la IA, hay grandes dilemas que emergen, no solamente en su aplicación en el campo militar, sino desde su fundamentación. ¿Es posible humanizar las acciones de las máquinas?, ¿son las acciones de la IA iguales a las humanas?, ¿existe una valoración moral en la IA?, ¿se pueden plasmar principios y virtudes en la IA?, ¿en quién recae la responsabilidad de las acciones de la IA?, ¿cómo diseñar, desarrollar, implementar y evaluar la IA con 
parámetros éticos?, ¿qué parámetros éticos debe tener la IA? Estas son algunas de las preguntas que se formulan en el desarrollo de esta investigación y que, al igual que desde la percepción de muchos de los autores que se analizaron, queda la sensación de que existe un vacío muy profundo sobre lo que puede llegar a ser la ética de la IA, entre otras cosas, como se comentaba al inicio del capítulo, por la ambigüedad y subjetividad del concepto.

Los análisis de la ética de la IA han girado en torno, desde el plano teórico, humanístico y conceptual, a las implicaciones sociales que tiene la implementación de nuevas tecnologías (Triana, 2017). En otro sentido, desde una visión práctica establecida por las ingenierías, se ha abogado a principios de diseño más específicos que permitan la trazabilidad de los algoritmos, la "explicabilidad", la rendición de cuentas y la supervisión humana (Marín, 2019, p. 11). Desde la intercepción multidisciplinar, se ha respaldado la confianza (e-trust) aplicada a contextos digitales (Ferrario et al., 2020, p. 528), pero también se ha contraargumentado esta posición, desde la psicología, manifestando que no se puede delegar confianza a la IA debido a que no son identificables estados emotivos o responsabilidades, requerimientos afectivos y normativos necesarios para confiar (Ryan, 2020, p. 2750)

En relación más específica con el concepto de ética, y sus alcances morales y legales, la Consejería Presidencial para Asuntos Económicos y Transformación Digital de Colombia (2020) consideró como ética de la IA "una rama de la ética que analiza y evalúa los dilemas morales que se derivan del despliegue de esta tecnología en la sociedad” (p. 14). Martínez Martínez (2019) la llevó a su interacción con los Derechos Humanos proponiendo que debe estar "centrada en la garantía de los derechos fundamentales" (p. 73), mientras Leslie (2019) logra una articulación de ambas propuestas formulando que debe ser un conjunto de valores, principios y técnicas que emplea estándares ampliamente aceptados de lo correcto y lo incorrecto para guiar la conducta de las máquinas (p. 3); precisamente, este último concepto es uno de los más constitutivos para el objetivo de la presente investigación. 


\section{Métodos}

Los resultados obtenidos en esta investigación corresponden a un nivel exploratorio y descriptivo. Según Arias (2012), esta delimitación del trabajo investigativo permite enmarcar el documento, por un lado, en objetos de investigación que requieren mayor reflexión científica para formular de manera más precisa sus problemas y, por otro, descripciones de esos objetos para establecer claridad y exactitud en su estructura. Al tener dos elementos de estudio, la IA y la ética, en específico la militar, el propósito metodológico fue, con base en la demarcación propuesta, concretar la posible correlación, no causal, entre estos dos escenarios para Colombia desde el contexto general global.

La pretensión de la investigación no fue dar explicaciones ni la aplicación práctica de los resultados. En ese sentido, no se tuvieron como orientación los postulados de los paradigmas positivista y sociocrítico, sino, de manera lógica con el problema de investigación, se reflexionó desde un paradigma interpretativo. Es decir, se buscó la comprensión y profundización del objeto de estudio desde un postulado ontológico múltiple y divergente que permitiera construir la exploración y posterior descripción, conservando factores subjetivos en la relación investigador-objeto.

Para lograr la aprehensión del problema con un método, técnica e instrumento, el ejercicio investigativo se generó con base en razonamientos cualitativos, adecuados para el paradigma propuesto. De esa forma, el principal método usado fue el análisis documental relacional desde el fundamento hermenéutico, entendido como aquel que permite "la búsqueda de sentido y de verdad [en] experiencias vitales y subjetivas" (Maldonado, 2016, p. 3) y, valorando esto, la fuente de información esencial fue secundaria, extraída de bases de datos bibliográficas y entidades públicas oficiales a nivel mundial.

Para la recolección y análisis de los documentos se siguieron cinco etapas, estructuradas con base en la propuesta de Ortiz Palanques (2016): (1) identificación de los temas que se quieren investigar (ética y ética militar, inteligencia artificial e inteligencia artificial y defensa); (2) planteamiento del objetivo sobre los temas (reflexionar de manera crítica sobre la información encontrada para el caso colombiano); (3) identificación de ideas principales y secundarias para 
cada documento; (4) preguntar y repreguntar (¡cómo el documento aporta a mi objetivo?) y (5) transmisión de los elementos encontrados. El procedimiento de esta técnica se soportó a través de una matriz de operacionalización de categorías y análisis documental, en la cual se plasmó la siguiente información para cada uno de los 75 documentos revisados: (1) la fuente y referencia; (2) la categoría o subcategoría; (3) al menos cuatro conceptos clave; (4) las ideas centrales; (5) citas textuales y (6) la forma de aplicación o correspondencia para la investigación.

Finalmente, para el tratamiento de los resultados encontrados en el instrumento se usó una unidad hermenéutica en el software Atlas.ti Versión 8. En esta herramienta se hizo la correspondencia de categorías y subcategorías como forma de codificación, con el fin de identificar las características más mencionadas y correlacionadas en cada caso, desde la orientación que se plantea con el problema y el diseño mismo de la investigación. En la tabla 1 se presenta la matriz realizada para el procesamiento propuesto en este software.

Tabla 1. Matriz de codificación para el procesamiento de datos en Atlas.ti 8

\begin{tabular}{|c|c|c|c|c|c|}
\hline Código & Nombre & Código & Nombre & Código & Nombre \\
\hline $\mathrm{C} 1$ & $\begin{array}{c}\text { inteligencia artificial } \\
\text { y ética }\end{array}$ & CA35 & human-in-the-loop & SC2 & governance \\
\hline $\mathrm{C} 2$ & $\begin{array}{c}\text { inteligencia artificial } \\
\text { y defensa }\end{array}$ & CA36 & buman-over-the-loop & SC3 & confianza \\
\hline $\mathrm{C} 3$ & $\begin{array}{l}\text { inteligencia artificial, } \\
\text { ética y defensa }\end{array}$ & CA37 & discriminación & SC4 & inclusión \\
\hline CE1 & $\begin{array}{l}\text { inteligencia artificial } \\
\text { y antropocentrismo }\end{array}$ & CA38 & auditoría & SC5 & accountability \\
\hline CE2 & $\begin{array}{l}\text { inteligencia artificial, } \\
\text { ética y medicina }\end{array}$ & CA39 & educación & SC6 & inequidad \\
\hline CE3 & $\begin{array}{c}\text { inteligencia artificial } \\
\text { y complejidad }\end{array}$ & CA40 & investigación & SC7 & principios \\
\hline CE4 & $\begin{array}{c}\text { inteligencia artificial, } \\
\text { ética y arte }\end{array}$ & CA41 & sesgo & SC8 & justicia \\
\hline CA1 & conceptual & CA42 & equidad & SC9 & datos \\
\hline
\end{tabular}




\begin{tabular}{|c|c|c|c|c|c|}
\hline Código & Nombre & Código & Nombre & Código & Nombre \\
\hline $\mathrm{CA} 2$ & machine learning & CA43 & decisión & SC10 & salud \\
\hline CA3 & autonomous systems & CA44 & viability & SC11 & algoritmos \\
\hline CA4 & situational awareness & CA45 & adaptabilidad & SC12 & decisiones \\
\hline CA5 & data fusion & CA46 & riesgo humano & $\mathrm{SC13}$ & efectividad \\
\hline CA6 & neural networks & CA47 & información & SC14 & riesgo \\
\hline CA7 & teaming & CA48 & armas & SC15 & seguridad \\
\hline CA8 & language processing & CA49 & reconocimiento & SC16 & estabilidad \\
\hline CA9 & training & CA50 & vehículos & SC17 & dilema \\
\hline CA10 & diseño & CA51 & dron & SC18 & innovación \\
\hline CA11 & desarrollo & CA52 & ciberseguridad & SC19 & militar \\
\hline CA12 & beneficence & CA53 & validación & SC20 & autónomo \\
\hline CA13 & non-malefincence & CA54 & dignidad & SC21 & competencia \\
\hline CA14 & autonomía & CA55 & sostenibilidad & SC22 & armas \\
\hline CA15 & justicia & CA56 & solidaridad & SC23 & ciberseguridad \\
\hline CA16 & explicability & CA57 & privacidad & SC24 & $\begin{array}{l}\text { machine } \\
\text { learning }\end{array}$ \\
\hline CA17 & confianza & CA58 & libertad & SC25 & social \\
\hline CA18 & inclusión & CA59 & beneficencia & SC26 & control \\
\hline CA19 & good & CA60 & inteligibilidad & SC27 & robótica \\
\hline CA20 & humanidad & CA61 & limitación & SC28 & derecho \\
\hline CA21 & responsabilidad & CA62 & traceability & SC29 & geopolítica \\
\hline CA22 & control & CA63 & armonía & SC30 & mejora \\
\hline CA23 & gobernanza & CA64 & datos & SC31 & filosofía \\
\hline CA24 & cooperación & CA65 & falsiability & SC32 & desafío \\
\hline CA25 & derecho & CA66 & competencia & SC33 & complejidad \\
\hline CA26 & seguridad & CA67 & vulnerabilidad & SC34 & valores \\
\hline CA27 & transparencia & CA68 & simulación & SC35 & estándar \\
\hline CA28 & cuidado & CA69 & guerra & SC36 & estrategia \\
\hline
\end{tabular}




\begin{tabular}{|c|c|c|c|c|c|}
\hline Código & Nombre & Código & Nombre & Código & Nombre \\
\hline CA29 & consentimiento & CA70 & integridad & SC37 & sensibilidad \\
\hline CA30 & $\begin{array}{l}\text { procesamiento de } \\
\text { lenguaje }\end{array}$ & CA71 & valor/moral & SC38 & robot \\
\hline CA31 & frabilidad & CA72 & predicción & SC39 & defensa \\
\hline CA32 & implementación & CE\# 1 & democracia & SC40 & políticas \\
\hline CA33 & evaluación & $\mathrm{CE} 2$ & dańo emocional & & \\
\hline CA34 & daño financiero & SC1 & ley & & \\
\hline \multicolumn{2}{|c|}{$\begin{array}{l}\mathrm{C} \rightarrow \text { Categoría } \\
\mathrm{SC} \rightarrow \text { Subcategoría } \\
\mathrm{CA} \rightarrow \text { Característica }\end{array}$} & & \multicolumn{2}{|c|}{$\begin{array}{l}\mathrm{CE} \rightarrow \text { Categoría emergente } \\
\mathrm{CE} \# \rightarrow \text { Característica emergente }\end{array}$} & \\
\hline
\end{tabular}

Fuente: elaboración propia con base en la matriz de operacionalización de categorías y análisis documental.

\section{Resultados}

\section{Por enraizamiento}

La categoría C1 (inteligencia artificial y ética) fue la que generó un mayor enraizamiento en el análisis (38), seguida por la categoría C2 (26) y C3 (10). A continuación, en la tabla 2 se presenta la relación del enraizamiento por categoría.

Tabla 2. Relación del enraizamiento por categoría

\begin{tabular}{cc}
\hline Código & Enraizamiento \\
\hline C1 & 38 \\
C2 & 26 \\
C3 & 10 \\
CE3 & 1 \\
CE1 & 1 \\
CE2 & 1 \\
CE4 & 1 \\
\hline
\end{tabular}

Fuente: elaboración propia con base en el análisis realizado en Atlas.ti 8. 
Solo cuatro subcategorías representaron un enraizamiento superior a 10 : SC19 (18), SC22 (14), SC23 (12) y SC15 (11), y doce entre 9 y 5: 9 (SC28 y SC9); 7 (SC39); 6 (SC20, SC12, SC14, SC24 y SC27); 5 (SC10, SC2, SC11 y SC7). Militar fue la subcategoría que más enraizamiento tuvo, como se representa en la tabla 3.

Tabla 3. Relación del enraizamiento por subcategoría

\begin{tabular}{lccc}
\hline Código & Enraizamiento & Código & Enraizamiento \\
\hline SC19 & 18 & SC5 & 3 \\
SC22 & 14 & SC30 & 2 \\
SC23 & 12 & SC36 & 2 \\
SC15 & 11 & SC25 & 2 \\
SC28 & 9 & SC3 & 2 \\
SC9 & 9 & SC34 & 2 \\
SC39 & 7 & SC33 & 2 \\
SC20 & 6 & SC31 & 2 \\
SC12 & 6 & SC6 & 1 \\
SC14 & 6 & SC17 & 1 \\
SC24 & 6 & SC4 & 1 \\
SC27 & 6 & SC16 & 1 \\
SC10 & 5 & SC8 & 1 \\
SC2 & 5 & SC37 & 1 \\
SC11 & 5 & SC21 & 1 \\
SC7 & 5 & SC35 & 1 \\
SC26 & 4 & SC32 & 1 \\
SC40 & 4 & SC38 & 1 \\
SC18 & 3 & SC29 & 1 \\
SC1 & 3 & SC13 & 1 \\
\hline
\end{tabular}

Fuente: elaboración propia con base en el análisis realizado en Atlas.ti 8. 
La característica CA21 tuvo el enraizamiento más alto (56), seguida por CA64 (47), CA11 (45), CA71 y CA26 (44). En la tabla 4 se presentan los resultados por característica.

Tabla 4. Relación del enraizamiento por característica

\begin{tabular}{|c|c|c|c|c|c|}
\hline Código & Enraizamiento & Código & Enraizamiento & Código & Enraizamiento \\
\hline CA21 & 56 & CA55 & 12 & CA66 & 3 \\
\hline CA64 & 47 & CA49 & 11 & $\mathrm{CE} \# 1$ & 3 \\
\hline CA11 & 45 & CA23 & 11 & CA33 & 3 \\
\hline CA71 & 44 & CA19 & 11 & CA7 & 2 \\
\hline CA26 & 44 & CA37 & 10 & CA35 & 2 \\
\hline CA57 & 36 & CA2 & 9 & CA9 & 2 \\
\hline CA48 & 36 & CA39 & 8 & CA61 & 1 \\
\hline CA22 & 35 & CA18 & 8 & CA36 & 1 \\
\hline CA25 & 34 & CA72 & 7 & CA31 & 1 \\
\hline CA15 & 32 & CA51 & 6 & CA38 & 1 \\
\hline CA52 & 31 & CA50 & 5 & CA44 & 1 \\
\hline CA46 & 31 & CA24 & 5 & CA13 & 1 \\
\hline CA27 & 28 & CA28 & 5 & CA8 & 1 \\
\hline CA43 & 27 & CA58 & 5 & CA5 & 1 \\
\hline CA69 & 25 & CA45 & 4 & CA59 & 1 \\
\hline CA10 & 24 & CA3 & 4 & CA6 & 1 \\
\hline CA17 & 21 & CA20 & 4 & CA4 & 1 \\
\hline CA14 & 20 & CA67 & 4 & CA65 & 1 \\
\hline CA16 & 20 & CA12 & 4 & CA70 & 1 \\
\hline CA40 & 17 & CA62 & 4 & CA30 & 1 \\
\hline CA42 & 15 & CA53 & 3 & $\mathrm{CE \# 2}$ & 1 \\
\hline CA32 & 14 & CA68 & 3 & CA60 & 1 \\
\hline CA47 & 13 & CA63 & 3 & CA1 & 1 \\
\hline CA41 & 13 & CA56 & 3 & & \\
\hline CA54 & 12 & CA29 & 3 & & \\
\hline
\end{tabular}

Fuente: elaboración propia con base en el análisis realizado en Atlas.ti 8. 


\section{Por densidad}

La categoría C1 (inteligencia artificial y ética) tiene la mayor densidad (22), seguida por la categoría C2 (20) y C3 (17). En la tabla 5 se presenta la relación de la densidad por categoría.

Tabla 5. Relación de densidad por categoría

\begin{tabular}{cc}
\hline Código & Densidad \\
\hline C1 & 22 \\
C2 & 20 \\
C3 & 17 \\
CE1 & 2 \\
CE2 & 2 \\
CE4 & 2 \\
CE3 & 1 \\
\hline
\end{tabular}

Fuente: elaboración propia con base en el análisis realizado en Atlas.ti 8.

Solo tres subcategorías tuvieron una densidad por encima de 40: SC20 (47), SC19 (43) y SC26 (40); cinco estuvieron por encima de 30: SC28 (39), SC9 (36), SC27 (34), SC7 (33) y SC15 (31). Autónomo fue la subcategoría que más densidad generó, como se representa en la tabla 6 .

Tabla 6. Relación de densidad por subcategoría

\begin{tabular}{cccc}
\hline Código & Densidad & Código & Densidad \\
\hline SC20 & 47 & SC34 & 19 \\
SC19 & 43 & SC16 & 16 \\
SC26 & 40 & SC17 & 15 \\
SC28 & 39 & SC10 & 14 \\
SC9 & 36 & SC18 & 14 \\
SC27 & 34 & SC5 & 14 \\
\hline
\end{tabular}




\begin{tabular}{cccc}
\hline Código & Densidad & Código & Densidad \\
\hline SC7 & 33 & SC25 & 14 \\
SC15 & 31 & SC30 & 12 \\
SC22 & 28 & SC21 & 10 \\
SC12 & 27 & SC3 & 8 \\
SC24 & 26 & SC8 & 8 \\
SC36 & 26 & SC32 & 8 \\
SC39 & 24 & SC13 & 7 \\
SC23 & 23 & SC33 & 6 \\
SC14 & 23 & SC4 & 5 \\
SC2 & 23 & SC38 & 5 \\
SC40 & 23 & SC31 & 4 \\
SC35 & 22 & SC6 & 4 \\
SC11 & 20 & SC37 & 4 \\
SC1 & 19 & SC29 & 3 \\
\hline
\end{tabular}

Fuente: elaboración propia con base en el análisis realizado en Atlas.ti 8.

La característica CA11 tuvo la densidad más alta (33), seguida por CA26 (27), CA64 (26), CA22 (25), CA21 y CA14 (24). En la tabla 7 se presentan los resultados de densidad por característica.

Tabla 7. Relación de densidad por característica

\begin{tabular}{cccccc}
\hline Código & Densidad & Código & Densidad & Código & Densidad \\
\hline CA11 & 33 & CA19 & 13 & CA56 & 4 \\
CA26 & 27 & CA41 & 11 & CA33 & 4 \\
CA64 & 26 & CA51 & 11 & CA13 & 4 \\
CA22 & 25 & CA54 & 10 & CA61 & 3 \\
CA21 & 24 & CA23 & 10 & CA59 & 3 \\
\hline
\end{tabular}




\begin{tabular}{|c|c|c|c|c|c|}
\hline Código & Densidad & Código & Densidad & Código & Densidad \\
\hline CA14 & 24 & CA37 & 10 & CA70 & 3 \\
\hline CA71 & 22 & CA72 & 10 & CA35 & 2 \\
\hline CA48 & 22 & $\mathrm{CA} 2$ & 9 & CA38 & 2 \\
\hline CA40 & 18 & CA39 & 9 & CA44 & 2 \\
\hline CA32 & 18 & CA20 & 9 & CA8 & 2 \\
\hline CA55 & 18 & CA18 & 8 & $\mathrm{CE \# 2}$ & 2 \\
\hline CA25 & 17 & CA12 & 8 & CA60 & 2 \\
\hline CA46 & 17 & CA24 & 7 & CA53 & 1 \\
\hline CA17 & 17 & CA58 & 7 & CA68 & 1 \\
\hline CA47 & 17 & CA67 & 7 & CA63 & 1 \\
\hline CA49 & 17 & CE\#1 & 7 & CA7 & 1 \\
\hline CA57 & 16 & CA28 & 6 & CA9 & 1 \\
\hline CA15 & 16 & CA66 & 6 & CA36 & 1 \\
\hline CA52 & 15 & CA30 & 6 & CA31 & 1 \\
\hline CA43 & 15 & CA1 & 6 & CA5 & 1 \\
\hline CA69 & 15 & CA50 & 5 & CA6 & 1 \\
\hline CA10 & 15 & CA45 & 5 & CA4 & 1 \\
\hline CA16 & 15 & CA62 & 5 & CA65 & 1 \\
\hline CA27 & 14 & CA29 & 5 & & \\
\hline CA42 & 14 & CA3 & 4 & & \\
\hline
\end{tabular}

Fuente: elaboración propia con base en el análisis realizado en Atlas.ti 8.

Finalmente, en la figura 1 se representa la interacción en red entre las subcategorías y características de acuerdo con la relación encontrada en el análisis hermenéutico. 


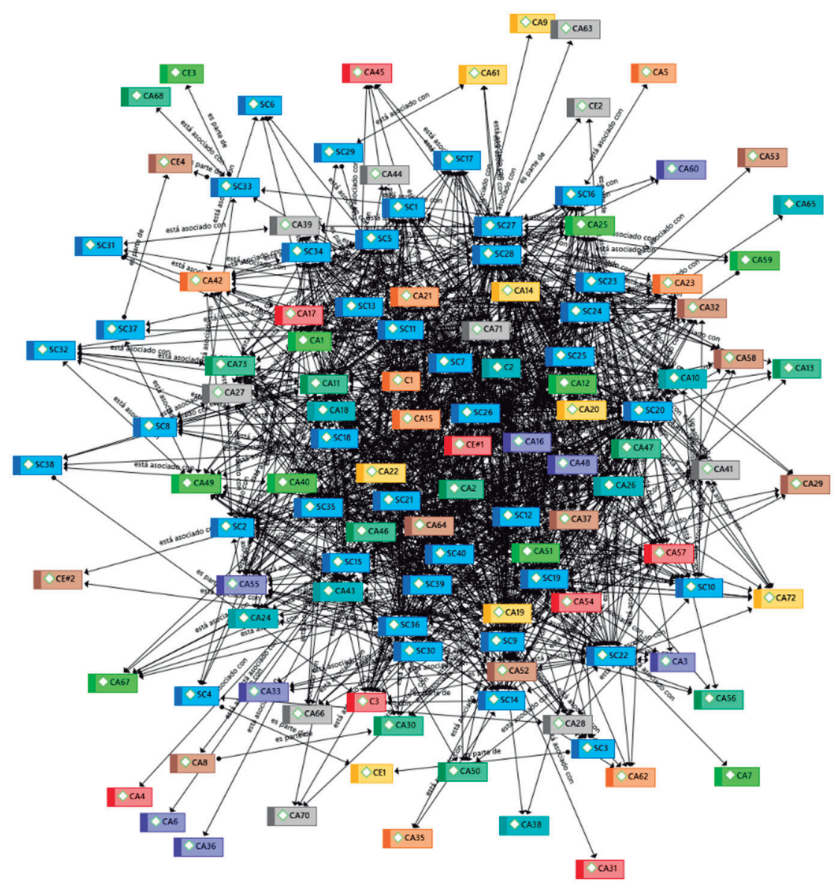

Figura 1. Interacción en red de subcategorías y características.

Fuente: elaboración propia con base en el análisis realizado en Atlas.ti 8.

\section{Discusión}

La principal categoría en el análisis documental realizado se enfoca en la relación exclusiva de la IA y la ética, resultado que era esperado, considerando que es el tema que más lleva discutiéndose en todo tipo de campo. Sin embargo, a pesar de lo anterior, hay relaciones importantes encontradas con ciertas subcategorías que se deben recalcar.

Por un lado, hay subcategorías que necesariamente y por su naturaleza forman parte de esta categoría, como la ley, los principios, la justicia, el derecho, la filosofía y los valores, pero, por otro lado, se identifican subcategorías que están impactando la formación de conocimiento y orientando los argumentos en relación con el tema desde esta visión. Governance, inequidad, social y politicas se vinculan, de manera directa o indirecta, con la necesidad de acción desde los Estados en la generación de marcos éticos para la inteligencia arti- 
ficial, tanto para los sistemas de implementación en desarrollos de gobierno digital, como para su regulación dentro de la sociedad. En otro sentido, hay una relación desde la ética y la IA en el plano estratégico y geopolítico que, en efecto, impacta al sector defensa; como argumenta Ortega Klein (2020), hay diferencias esenciales en los esfuerzos de actores internacionales importantes que se pueden percibir como sesgados por sus intereses, a pesar de compartir conceptualmente muchos de los principios propuestos.

Un sector importante de la academia se está enfocando en el análisis ético de la IA para el sector salud y, en general, las propuestas se encuentran encaminadas al diseño de algoritmos y procesos de machine learning, a la responsabilidad de las acciones, la autonomía de las máquinas y los avances en robótica. De manera frecuente se comenta sobre estándares y desafíos de estos, así como también se proponen principios y valores morales que, con base en una visión amplia, tienden a ser reiterativos en diferentes documentos, oficiales y académicos, como se presentará más adelante.

Ahora bien, en relación con la $I_{A}$ y la defensa, la mayor parte de la literatura se centra en describir o presentar los avances científicos y de innovación en la materia. La implementación de la IA en el campo militar y de la seguridad ha generado discusión sobre temas como las armas autónomas, los vehiculos no tripulados, la ciberseguridad automatizada, el mejoramiento de capacidades "clásicas", el fortalecimiento de capacidades espaciales, la robótica, entre otros. El análisis con subcategorías permitió evidenciar que hay asuntos que, aunque no se tratan de manera extensa, son comentados. El primero tiene correspondencia con la innovación, el tratamiento de datos y la implementación de algoritmos más seguros, controlables y confiables; un ejemplo de esto es el Proyecto XaI de Darpa en Estados Unidos (Turek, s. f.). El segundo guarda relación con los dilemas que se pueden presentar en estos escenarios y muestra la necesidad de decisiones efectivas y estables, e inclusive, en algunos casos, llega a evaluaciones en derechos (Derecho Internacional Humanitario y Derecho Internacional de los Derechos Humanos).

La categoría de inteligencia artificial, ética y defensa no representó una densidad significativa desde las subcategorías encontradas y analizadas, tampoco desde el enraizamiento; no obstante, es necesario describir al menos 
las siguientes tres relaciones encontradas en la codificación: (1) los pocos análisis que se han dado en esta rama específica del tema coinciden, casi siempre, en la exigencia de plasmar políticas, leyes y estándares que reflejen los principios y valores éticos requeridos para disminuir los riesgos en el uso de IA militar. (2) También hay autores que van más allá del "qué" al "cómo" y proponen modelos prácticos sobre los algoritmos para su implementación en las fases de diseño y desarrollo, en especial enfocadas en sistemas autónomos, sistemas de armas, robótica y machine learning. (3) Por último, hay gobiernos que tienen en sus objetivos estratégicos el desarrollo de ética para este campo de acción, como Francia y Estados Unidos, lo cual refleja la importancia de iniciativas estatales en política de seguridad nacional.

Las categorías emergentes resultan de suma importancia para la investigación por su aporte multidisciplinar y la propuesta de enfoques y perspectivas diferentes. La postura antropocentrista sugiere una visión de confianza e inclusión; la relacionada con la complejidad formula la posibilidad de simular, por medio de agentes computacionales, los dilemas morales y éticos de la IA; el arte pone de manifiesto el problema suscitado sobre los derechos de autor en la elaboración de piezas y, el más importante, la medicina presenta disyuntivas similares al marco militar por tratarse, en algunas ocasiones, del derecho fundamental a la vida. Al respecto, Ho (2019) argumenta, desde un análisis en la medicina, que los sistemas de machine learning no son $100 \%$ matemáticos o de valores neutrales, sino que son creados con fallas, sesgos humanos y prejuicios (p. 36).

Centrando el análisis ahora en un nivel específico, se debe iniciar con la característica que más densidad obtuvo en el ejercicio: el desarrollo. Como se comentó anteriormente, la IA tiene al menos cuatro fases: diseño, desarrollo, implementación y evaluación. En la generalidad de la documentación revisada, las primeras dos fases son las más importantes y en donde se debe intervenir "de manera ética" la IA; muestra de esto es la densidad que resulta del ejercicio. Como argumenta Martin (2019), "el desarrollo de algoritmos es moralmente relevante en términos de no solo crear errores, sino también en delegar tareas de quién puede y debe identificar, juzgar y corregir errores en decisiones algorítmicas" (p. 140), así que, claramente, es el proceso más crítico y, por la misma razón, en el que se ha enfocado gran parte de las propuestas. 
Otra característica relevante que emerge de este trabajo es la seguridad, desde tres sentidos. Es claro, como se ha enunciado, que la Ia funciona por medio de procesos algorítmicos sobre datos que permiten el comportamiento, la decisión o la acción de una u otra manera. Así, la seguridad de los datos, pero también de la integridad de los sistemas, es crucial para mantener las funcionalidades establecidas en una máquina. Con este concepto también se puede advertir, en otro sentido, la aplicación de IA en seguridad y, por ejemplo, de manera puntual, en ciberseguridad. Según Terrones Rodríguez (2018), "la industria militar es una de las grandes beneficiadas en la actualidad del avance de la IA, dado que las naciones más desarrolladas mantienen una carrera militar en la que la IA es su eje principal" (p. 149). Por otro lado, en menor medida, se identifica un debate vinculado a la seguridad que radica en la que es propia de los individuos: nos referimos con lo anterior al uso de IA para vulnerar desde datos personales hasta hábitos de consumo.

En relación con los principios y valores identificados en la revisión documental, a continuación se exponen los que se originaron en la generalidad, iniciando por la equidad. Orr y Davis (2020) presentan un estudio enfocado en la ética de la IA desde la inequidad, el cual revela una relación desequilibrada entre los que imponen los estándares y los que los implementan, "uno de poder y el otro de experticia técnica" (p. 725). Al menos tres propuestas contienen esta característica dentro de la revisión realizada: UK House of Lords, World Commission on the Ethics of Scientific Knowledge and Technology - COMEsT y EURON. La gobernanza guarda correspondencia de manera directa e indirecta con este punto, siendo China uno de los Estados que más se acerca al concepto. La definición de políticas sobre IA necesariamente debe tener como prioridad la gobernanza social y moral; no solo políticas estatales, sino, del mismo modo, comerciales, educativas y militares. La gobernanza de la Ia puede ser entendida como una forma de control: "Mientras los errores pueden ser inadvertidos, las decisiones de gobernanza no. La falta de intencionalidad puede ser una excusa justa para un error, pero no para no gobernar errores" (Martin, 2019, p. 132).

La no-maleficencia es uno de los aportes realizados por Ai4people sobre los principios de la IA. Floridi et al. (2018) lo enmarcan en su relación con otros tres: privacidad, seguridad y precaución sobre la capacidad. Asimismo, 
la beneficencia como principio apoya al anterior desde tres posibilidades: la promoción de bienestar, preservar la dignidad y sostener el planeta; mientras el denominado explicabilty permite habilitar a los demás principios mediante la inteligibilidad y la responsabilidad. La validación, como se sugiere en algunos documentos, guarda concordancia con la explicabilidad, la predicción y la transparencia, siendo, en un sentido práctico, a lo que apuntan los autores que abogan por la configuración ética desde la fase de desarrollo.

En un plano legal, ciertos esfuerzos se han encauzado al refuerzo de derechos fundamentales que pueden considerarse en riesgo, como la privacidad, la libertad, la humanidad y la dignidad. En el caso estadounidense se están llevando a cabo investigaciones desde el derecho, las ciencias sociales y la ética, incluyendo la privacidad, los derechos civiles y las libertades civiles, así como la experiencia específica y ciertos dominios de aplicación (Executive Office of the President of the United States, 2019, p. 18). Finalmente, un principio diferencial que se identificó es la armonía y cooperación: "La armonía debe ser considerada como un principio adicional de la ética de la IA por tres razones: ofrece una perspectiva valiosa en el contexto de sistemas de IA, no está inmersa en otro principio y no relega otros principios" (Berberich et al., 2020).

\section{Conclusión}

En primer lugar, las reflexiones que se realizan en el mundo sobre los límites éticos de la inteligencia artificial son diversas y pueden abarcar, desde perspectivas y disciplinas, todos los campos de la vida humana. No obstante, la literatura se ha enfocado específicamente en dos cuestiones esenciales: el qué y el cómo. Resulta necesario el abordaje de ambas preguntas de forma articulada e interdisciplinar, pues, sin pretender hacer generalizaciones, la ciencia y la sociedad se necesitan de forma bidireccional, considerando que la primera es importante para el desarrollo de la segunda, pero esta también debe corresponder a las estructuras éticas, morales y legales que regulan la vida en comunidad. Con lo anterior no se pretende decir que a la ciencia le pertenezca el cómo y a la sociedad el qué, por el contrario, cada uno se debe estudiar desde la unión de las dos perspectivas con el fin de fijar puntos de 
encuentro, metas, cuestionamientos e intereses comunes, reales y alcanzables, teniendo, por ejemplo, orientaciones desde categorías emergentes como la IA y la complejidad.

En segundo lugar, considerando lo anterior, resulta fundamental establecer los marcos de acción de la IA en la seguridad y defensa del país. Es claro que para algunos países del mundo es un tema avanzado que forma parte de sus sistemas de investigación e innovación, sin embargo, en el caso colombiano se debe, aparte de revisar las experiencias internacionales, fijar proyecciones que engloben la innovación y el desarrollo y, de manera paralela, lineamientos, políticas o manuales éticos para cada una de las fases de la IA en este campo y para todos los niveles. También, en otro sentido, es importante poner en consideración una subcategoría que afecta de manera directa a los Estados y que, desde el punto de vista estratégico, no ha sido estudiada en profundidad: la geopolítica. La definición de una "carrera armamentista”, con la IA como core, genera de forma ineludible un interés nacional desde la seguridad y la defensa planificado hacia el futuro.

Por último, en tercer lugar, el trabajo conjunto entre las ciencias formales y no formales tiene que proponer al menos tres elementos orientadores: (1) la articulación y conceptualización de principios como la armonía, la predicción, la aplicabilidad, la gobernanza, la inteligibilidad, entre otros contemplados como subcategorías en la presente investigación, con manuales y estructuras operacionales de las Fuerzas Militares. (2) El concepto de ética militar debe ser replanteado de tal manera que no solo abarque la condición humana sino, más allá, también su extensión sobre la responsabilidad en decisiones y sesgos de cualquier etapa de la IA. Finalmente, (3) esa estructura fundante que permiten los dos primeros puntos debe dar lugar a consideraciones legales que, aunque responden a condiciones éticas y morales, se enfocan de manera específica en el Derecho Internacional de los Derechos Humanos y el Derecho Internacional Humanitario, es decir, con lo anterior, a la protección de derechos fundamentales y al cumplimiento de principios del ius in bellum. 


\section{Referencias}

Arias, F. G. (2012). El proyecto de investigación: Introducción a la metodología científica (6. ${ }^{\mathrm{a}}$ ed.). Editorial Episteme.

Berberich, N., Nishida, T., \& Suzuki, S. (2020). Harmonizing artificial intelligence for social good. Philosophy \& Technology, (33), 613-638 https://doi.org/10.1007/s13347-02000421-8

Betancur Jiménez, G. E. (2016). La ética y la moral: Paradojas del ser humano. CES Psicología, 9(1), 109-121. https://doi.org/10.21615/cesp.9.1.7

Consejería Presidencial para Asuntos Económicos y Transformación Digital de Colombia. (2020). Marco ético para la inteligencia artificial en Colombia (Documento para Discusión). Gobierno de Colombia.

Consejo Nacional de Política Económica y Social (CONPES) 3995. (2020). Política Nacional de Confianza y Seguridad Digital. Departamento Nacional de Planeación. https://colaboracion.dnp.gov.co/CDT/Conpes/Econ\%C3\%B3micos/3995.pdf

De Swarte, T., Boufous, O., \& Escalle, P. (2019). Artificial intelligence, ethics and human values: The cases of military drones and companion robots. Artificial Life and Robotics, 24(3), 291-296. https://doi.org/10.1007/s10015-019-00525-1

Defense Innovation Board. (2020). AI principles: Recommendations on the ethical use of artificial intelligence by the Department of Defense. https://media.defense.gov/2019/ Oct/31/2002204458/-1/-1/0/DIB_AI_PRINCIPLES_PRIMARY_DOCUMENT.PDF

Executive Office of the President of the United States. (2019). 2016 - 2019 Progress Report: Advancing Artificial Intelligence R\&D. 48. https://www.nitrd.gov/pubs/AI-Researchand-Development-Progress-Report-2016-2019.pdf

Ferrario, A., Loi, M., \& Viganò, E. (2020). In AI we trust incrementally: A multi-layer model of trust to analyze human-artificial intelligence interactions. Philosophy \& Technology, 33(3), 523-539. https://doi.org/10.1007/s13347-019-00378-3

Floridi, L., Cowls, J., Beltrametti, M., Chatila, R., Chazerand, P., Dignum, V., Luetge, C., Madelin, R., Pagallo, U., Rossi, F., Schafer, B., Valcke, P., \& Vayena, E. (2018). AI4People - An ethical framework for a good AI society: Opportunities, risks, principles, and recommendations. Minds and Machines, 28(4), 689-707. https://doi.org/10.1007/s11023018-9482-5

Fuerzas Militares de Colombia. (2016). Manual de generalidades éticas para la vocación militar. Imprenta y Publicaciones de las Fuerzas Militares. https://www.mindefensa.gov. co/irj/go/km/docs/Mindefensa/Documentos/descargas/Sobre_el_Ministerio/Control_ Interno/documentos/manual_generalidades_eticas_FFMM.pdf

Ho, A. (2019). Deep ethical learning: Taking the interplay of human and artificial intelligence seriously. Hastings Center Report, 49(1), 36-39. https://doi.org/10.1002/hast.977

Jensen, B. M., Whyte, C., \& Cuomo, S. (2020). Algorithms at war: The promise, peril, and limits of artificial intelligence. International Studies Review, 22(3), 526-550. https:// doi.org/10.1093/isr/viz025 
Leslie, D. (2019, junio 11). Understanding artificial intelligence ethics and safety: A guide for the responsible design and implementation of AI systems in the public sector. https://doi. org/10.5281/ZENODO.3240529

Maldonado Oñate, R. (2016). El método hermenéutico en la investigación cualitativa. https:// doi.org/10.13140/RG.2.1.3368.5363

Marín García, S. (2019). Ética e inteligencia artificial. Servicio de Publicaciones de la Universidad de Navarra. https://doi.org/10.15581/018.ST-522

Martin, K. (2019). Designing ethical algorithms. MIS Quarterly Executive, (18), 129-142. https://doi.org/10.17705/2msqe.00012

Martínez Martínez, R. (2019). Inteligencia artificial desde el diseño. Retos y estrategias para el cumplimiento normativo. Revista Catalana de Dret Públic, (58), 64-81. https://doi. org/10.2436/rcdp.i58.2019.3317

Orr, W., \& Davis, J. L. (2020). Attributions of ethical responsibility by artificial intelligence practitioners. Information, Communication \& Society, 23(5), 719-735. https://doi. org/10.1080/1369118X.2020.1713842

Ortega Klein, A. (2020). Geopolítica de la ética en inteligencia artificial. Real Instituto Elcano.

Ortiz Palanques, M. (2016). Metodología y técnica hermenéutica.

Paredes Hernández, E., \& Velasco Espitia, M. E. (2014). Ética. Universidad de Pamplona. http:// www.unipamplona.edu.co/unipamplona/portalIG/home_109/recursos/octubre2014/ administraciondeempresas/semestre5/11092015/eticaadmin.pdf

Real Academia Española. (2019). Ético, ca. https://dle.rae.es/\%C3\%A9tico

Robles Carrillo, M. (2020). Artificial intelligence: From ethics to law. Telecommunications Policy, 44(6), 101-937. https://doi.org/10.1016/j.telpol.2020.101937

Ryan, M. (2020). In AI we trust: Ethics, artificial intelligence, and reliability. Science and Engineering Ethics, 26(5), 2749-2767. https://doi.org/10.1007/s11948-020-00228-y

Terrones Rodríguez, A. L. (2018). Inteligencia artificial y ética de la responsabilidad. Cuestiones de Filosofía, 4(22), 141-170. https://doi.org/10.19053/01235095.v4.n22.2018.8311

Triana Tacuma, E. (2017). Ética informática. Daena: International Journal of Good Conscience, 12(3), 272-279.

Turek, M. (s. f.). Explainable Artificial Intelligence (XAI). https://www.darpa.mil/program/ explainable-artificial-intelligence

\section{Bibliografía usada para la matriz de análisis}

Allen, G., \& Chan, T. (2017). Artificial Intelligence and National Security. Belfer Center for Science and International Affairs. https://www.belfercenter.org/sites/default/files/files/ publication/AI\%20NatSec\%20-\%20final.pdf

Aznarte, J. L. (2020). Consideraciones éticas en torno al uso de tecnologías basadas en datos masivos en la UNED. RIED. Revista Iberoamericana de Educación a Distancia, 23(2), 237. https://doi.org/10.5944/ried.23.2.26590 
Beijing Academy of Artificial Intelligence. (2019). Beijing AI Principles. https://www.baai.ac.cn/ news/beijing-ai-principles-en.html

Berberich, N., Nishida, T., \& Suzuki, S. (2020). Harmonizing artificial intelligence for social good. Philosophy \& Technology, (33), 613-638. https://doi.org/10.1007/s13347-02000421-8

Consejería Presidencial para Asuntos Económicos y Transformación Digital de Colombia. (2020). Marco ético para la inteligencia artificial en Colombia (Documento para Discusión). Gobierno de Colombia.

Cotino Hueso, L. (2019). Ética en el diseño para el desarrollo de una inteligencia artificial, robótica y big data confiables y su utilidad desde el derecho. Revista Catalana de Dret Públic, (58), 29-48.

Crosby, C. (2020). Operationalizing artificial intelligence for algorithmic warfare. Military Review, (11), 43-51. https://www.armyupress.army.mil/Portals/7/military-review/ Archives/English/JA-20/Crosby-Operationalizing-AI-1.pdf

Davis, Z. S. (2019). Artificial intelligence on the battlefield. Center for Global Security Research Lawrence Livermore National Laboratory.

De Swarte, T., Boufous, O., \& Escalle, P. (2019). Artificial intelligence, ethics and human values: The cases of military drones and companion robots. Artificial Life and Robotics, 24(3), 291-296. https://doi.org/10.1007/s10015-019-00525-1

Defense Innovation Board. (2020). AI principles: Recommendations on the ethical use of artificial intelligence by the Department of Defense. https://media.defense.gov/2019/ Oct/31/2002204458/-1/-1/0/DIB_AI_PRINCIPLES_PRIMARY_DOCUMENT.PDF

European Commission for the Efficiency of Justice. (2019). Council of Europe adopts first European Ethical Charter on the use of artificial intelligence in judicial systems. CEPEJ European Ethical Charter on the Use of Artificial Intelligence (AI) in Judicial Systems and Their Environment. https://www.coe.int/en/web/cepej/cepej-european-ethical-charter-on-the-use-of-artificial-intelligence-ai-in-judicial-systems-and-their-environment

European Parliamentary Research Service. (2020). The ethics of artificial intelligence: Issues and initiatives. Publications Office. https://data.europa.eu/doi/10.2861/6644

Executive Office of the President of the United States. (2019). 2016-2019. Progress Report: Advancing Artificial Intelligence R\&D. 48. https://www.nitrd.gov/pubs/AI-Researchand-Development-Progress-Report-2016-2019.pdf

Farisco, M., Evers, K., \& Salles, A. (2020). Towards establishing criteria for the ethical analysis of artificial intelligence. Science and Engineering Ethics, 26(5), 2413-2425. https://doi. org/10.1007/s11948-020-00238-w

Ferrario, A., Loi, M., \& Viganò, E. (2020). In AI we trust incrementally: A multi-layer model of trust to analyze human-artificial intelligence interactions. Philosophy \& Technology, 33(3), 523-539. https://doi.org/10.1007/s13347-019-00378-3

Floridi, L., Cowls, J., Beltrametti, M., Chatila, R., Chazerand, P., Dignum, V., Luetge, C., Madelin, R., Pagallo, U., Rossi, F., Schafer, B., Valcke, P., \& Vayena, E. (2018). AI4People - An ethical framework for a good AI society: Opportunities, risks, principles, and recom- 
mendations. Minds and Machines, 28(4), 689-707. https://doi.org/10.1007/s11023018-9482-5

Floridi, L., Cowls, J., King, T. C., \& Taddeo, M. (2020). How to design AI for social good: Seven essential factors. Science and Engineering Ethics, 26(3), 1771-1796. https://doi. org/10.1007/s11948-020-00213-5

G7 Innovation Ministers. (2018). Statement on Artificial Intelligence. www.g8.utoronto.ca/ employment/2018-labour-annex-b-en.html

Galanter, P. (2020). Towards ethical relationships with machines that make art. Artnodes, (26). https://doi.org/10.7238/a.v0i26.3371

Gesley, J. (2019). Regulation of artificial intelligence in selected jurisdictions. The Law Library of Congress US.

Golden, P. E. (2020). DoDs artificial intelligence problem. Army Law.

Gómez de Ágreda, Á. (2020). Ethics of autonomous weapons systems and its applicability to any AI systems. Telecommunications Policy, 44(6), 101953. https://doi.org/10.1016/j. telpol.2020.101953

Gordon-Murnane, L. (2018). Ethical, Explainable Artificial Intelligence. Online Searcher Information Discovery, Technology, Strategies, 42(2), 22-30.

Goztepe, K., Dizdaro, V., \& Sağıroğlu, Ş. (2015). New directions in military and security studies. International Journal of Information Security Science, 4(2), 69-80.

Hagendorff, T. (2020). The ethics of AI ethics: An evaluation of guidelines. Minds and Machines, 30(1), 99-120. https://doi.org/10.1007/s11023-020-09517-8

Hernández-Pérez, T. (2018). Ética y maximalismo digital: Necesidad del pensamiento crítico sobre las tecnologías. Anuario ThinkEPI, (12), 352-355. https://doi.org/10.3145/ thinkepi.2018.59

Ho, A. (2019). Deep ethical learning: Taking the interplay of human and artificial intelligence seriously. Hastings Center Report, 49(1), 36-39. https://doi.org/10.1002/hast.977

Hoadley, D. S., \& Lucas, N. J. (2018). Artificial Intelligence and National Security. Congressional Research Service, (42). https://digital.library.unt.edu/ark:/67531/metadc1157028/

Horowitz, M. C., Kahn, L., \& Mahoney, C. (2020). The future of military applications of artificial intelligence: A role for confidence-building measures? Orbis, 64(4), 528-543. https:// doi.org/10.1016/j.orbis.2020.08.003

IBM Design Program Office. (2019). Everyday ethics for artificial intelligence. IBM. https://www. $\mathrm{ibm} . \mathrm{com} /$ watson/assets/duo/pdf/everydayethics.pdf

Jensen, B. M., Whyte, C., \& Cuomo, S. (2020). Algorithms at war: The promise, peril, and limits of artificial intelligence. International Studies Review, 22(3), 526-550. https:// doi.org/10.1093/isr/viz025

Johnson, J. S. (2020). Artificial intelligence: A threat to strategic stability. Artificial Intelligence, Strategic Studies Quarterly Spring, 16-39. https://www.airuniversity.af.edu/Portals/10/ SSQ/documents/Volume-14_Issue-1/Johnson.pdf

Kabbas, A., Alharthi, A., \& Munshi, A. (2020). Artificial Intelligence applications in cybersecurity. International Journal of Computer Science and Network Security, 20(2), 120-124. 
Kirk, A. D. (2019). Artificial Intelligence and the Fifth Domain. U.S. Air Force Academy, Department of Law, (80), 183-235.

Leslie, D. (2019). Understanding artificial intelligence ethics and safety: A guide for the responsible design and implementation of AI systems in the public sector. Zenodo. https://doi. org/10.5281/ZENODO.3240529

Maas, M. M. (2019). How viable is international arms control for military artificial intelligence? Three lessons from nuclear weapons. Contemporary Security Policy, 40(3), 285-311. https://doi.org/10.1080/13523260.2019.1576464

Marín García, S. (2019). Ética e inteligencia artificial. Servicio de Publicaciones de la Universidad de Navarra. https://doi.org/10.15581/018.ST-522

Martin, K. (2019). Designing Ethical Algorithms. MIS Quarterly Executive, 18(2), 129-142. https://doi.org/10.17705/2msqe.00012

Martínez Díaz, R., \& Zapata Domínguez, Á. (2014). Las ciencias sociales y los dispositivos de la complejidad. Cuadernos de Administración, 29(50), 123-131. https://doi. org/10.25100/cdea.v29i50.48

Martínez Martínez, R. (2019). Inteligencia artificial desde el diseño. Retos y estrategias para el cumplimiento normativo. Revista Catalana de Dret Públic, (58), 64-81. https://doi. org/10.2436/rcdp.i58.2019.3317

Meziat, C., \& Guille, L. (2019). Protecting tomorrows world today. Wavestone - Financial Communication Cybermaturity Index, (12). https:/www.wavestone.com/app/ uploads/2019/09/Wavestone_2019_AI_Cybersecurity_English.pdf

Ministère des Armées. (2019). Artificial Intelligence in Support of Defence. AI Task Force, République Française. https://www.defense.gouv.fr/content/download/573877/9834690/ Strat\%C3\%A9gie\%20de\%201\%27IA-UK_9\%201\%202020.pdf

Montes, G. A., \& Goertzel, B. (2019). Distributed, decentralized, and democratized artificial intelligence. Technological Forecasting and Social Change, (141), 354-358. https://doi. org/10.1016/j.techfore.2018.11.010

Morgan, F. E., Boudreaux, B., Lohn, A. J., Ashby, M., Curriden, C., Klima, K., \& Grossman, D. (2020). Military applications of artificial intelligence: Ethical concerns in an uncertain world. Rand Corporation. https://www.rand.org/pubs/research_reports/RR3139-1.html

Mori, S. (2018). US Defense Innovation and Artificial Intelligence. Asia-Pacific Review, 25(2), 16-44. https://doi.org/10.1080/13439006.2018.1545488

Morley, J., Floridi, L., Kinsey, L., \& Elhalal, A. (2020). From what to how: An initial review of publicly available ai ethics tools, methods and research to translate principles into practices. Science and Engineering Ethics, 26(4), 2141-2168. https://doi.org/10.1007/ s11948-019-00165-5

Moy, G. (2020). Recentadvances in artificial intelligence and their impacton defence. https://www. dst.defence.gov.au/sites/default/files/publications/documents/DST-Group-TR-3716_0.pdf

NATO Science, \& Technology Organization. (2020). Science \& Technology Trends 2020-2040. https://www.nato.int/nato_static_fl2014/assets/pdf/2020/4/pdf/190422-ST_Tech_Trends_ Report_2020-2040.pdf 
Novais, P., \& Freitas, P. M. (2018). Inteligência Artificial e Regulação de algoritmos. Ministério da Ciência, Tecnologia, Inovaçóes e Comunicações.

NSTC MLAI, \& NSTC NITRD. (2020). AI and cybersecurity: opportunities and challenges. https://www.nitrd.gov/pubs/AI-CS-Tech-Summary-2020.pdf

Recommendation of the Council on Artificial Intelligence "intergovernmental standard on AI", 0449 OECD/LEGAL/0449. (2020). http://legalinstruments.oecd.org

O'Hanlon, M. (2020). Forecasting change in military technology, 2020-2040. https://www. brookings.edu/research/forecasting-change-in-military-technology-2020-2040/

Orr, W., \& Davis, J. L. (2020). Attributions of ethical responsibility by Artificial Intelligence practitioners. Information, Communication \& Society, 23(5), 719-735. https://doi. org/10.1080/1369118X.2020.1713842

Ortega Klein, A. (2020). Geopolitica de la ética en inteligencia artificial. Real Instituto Elcano.

Pfaff, C. A. (2019). The ethics of acquiring disruptive technologies: Artificial intelligence, autonomous weapons, and decision support systems. Simons Center Special Report, Ethical Implications of Large Scale Combat Operations, (3)17. https://thesimonscenter.org/ wp-content/uploads/2019/10/Ethics-Symp-2019-p129-156.pdf

Prabhu, S. P. (2019). Ethical challenges of machine learning and deep learning algorithms. The Lancet Oncology, 20(5), 621-622. https://doi.org/10.1016/S1470-2045(19)30230-X

Rabadán, A. T. (2019). Horizonte de la inteligencia artificial y neurociencias. Acerca de robots, androides y cyborgs. Medicina, (79), 397-400. https://www.medicinabuenosaires.com/indices-de-2010-a-2019/volumen-79-ano-2019-no-5-indice/horizonte/

Robles Carrillo, M. (2020). Artificial intelligence: From ethics to law. Telecommunications Policy, 44(6), 101937. https://doi.org/10.1016/j.telpol.2020.101937

Ryan, M. (2020). In AI we trust: Ethics, artificial intelligence, and reliability. Science and Engineering Ethics, 26(5), 2749-2767. https://doi.org/10.1007/s11948-020-00228-y

Safdar, N. M., Banja, J. D., \& Meltzer, C. C. (2020). Ethical considerations in artificial intelligence. European Journal of Radiology, (122). https://doi.org/10.1016/j. ejrad.2019.108768

Sanders, T. (2020). Testing the black box: Institutional investors, risk disclosure, and ethical AI. Philosophy \& Technology. https://doi.org/10.1007/s13347-020-00409-4

Schwitzgebel, E., \& Garza, M. (2015). A defense of the rights of artificial intelligences: Defense of the rights of artificial intelligences. Midwest Studies in Philosophy, 39(1), 98-119. https://doi.org/10.1111/misp.12032

Sharikov, P. (2018). Artificial intelligence, cyberattack, and nuclear weapons - A dangerous combination. Bulletin of the Atomic Scientists, 74(6), 368-373. https://doi.org/10.108 0/00963402.2018.1533185

Terrones Rodríguez, A. L. (2018). Inteligencia artificial y ética de la responsabilidad. Cuestiones de Filosofía, 4(22), 141-170. https://doi.org/10.19053/01235095.v4.n22.2018.8311

Triana Tacuma, E. (2017). Ética informática. Daena: International Journal of Good Conscience, 12(3), 272-279. 
Turchin, A., \& Denkenberger, D. (2020). Classification of global catastrophic risks connected with artificial intelligence. AI \& Society, 35(1), 147-163. https://doi.org/10.1007/ s00146-018-0845-5

Turek, M. (s. f.). Explainable Artificial Intelligence (XAI). https://www.darpa.mil/program/ explainable-artificial-intelligence

United Nations Interregional Crime and Justice Research Institute, \& The International Criminal Police Organization. (2020). Towards Responsible AI Innovation. http://www. unicri.it/towards-responsible-artificial-intelligence-innovation

United Nations, \& Stanley Center for Peace and Security. (2019). The Militarization of Artificial Intelligence. https://stanleycenter.org/wp-content/uploads/2020/06/TheMilitarizationArtificialIntelligence.pdf

US Department of Defense. (2020). DOD adopts ethical principles for artificial intelligence. https://www.defense.gov/Newsroom/Releases/Release/Article/2091996/dod-adopts-ethical-principles-for-artificial-intelligencel

US Department of Homeland Security. (2018). Using standards to mitigate risks: Ai. https:// www.dhs.gov/sites/default/files/publications/2018_AEP_Artificial_Intelligence.pdf

Wang, Y., Friyia, D., Liu, K., \& Cohen, R. (2018). An Architecture for a Military AI System with Ethical Rules. https://www.aaai.org/ocs/index.php/SSS/SSS18/paper/view/17497/15379

Wasilow, S., \& Thorpe, J. B. (2019). Artificial intelligence, robotics, ethics, and the military: A Canadian perspective. AI Magazine, 40(1), 37-48. https://doi.org/10.1609/aimag. v40i1.2848

Wu, W., Huang, T., \& Gong, K. (2020). Ethical principles and governance technology development of AI in China. Engineering, 6(3), 302-309. https://doi.org/10.1016/j. eng.2019.12.015

Zouave, E., Gustafsson, T., Bruce, M., \& Colde, K. (2020). Artificially Intelligent Cyberattacks. https://www.statsvet.uu.se/digitalAssets/769/c_769530-1_3-k_rapport-foi-vt20.pdf 